\title{
Cerebral protection in type A aortic dissection
}

\author{
Christian V. Ghincea, Muhammad Aftab, Yuki Ikeno, Andrew L. Mesher, T. Brett Reece \\ Division of Cardiothoracic Surgery, Department of Surgery, University of Colorado School of Medicine, Anschutz Medical Campus, Aurora, CO, \\ USA \\ Contributions: (I) Conception and design: TB Reece, CV Ghincea, Y Ikeno, M Aftab; (II) Administrative support: TB Reece, AL Mesher, M Aftab; (III) \\ Provision of study materials or patients: TB Reece, M Aftab, AL Mesher; (IV) Collection and assembly of data: CV Ghincea, TB Reece, AL Mesher; \\ (V) Data analysis and interpretation: CV Ghincea, TB Reece; (VI) Manuscript writing: All authors; (VII) Final approval of manuscript: All authors. \\ Correspondence to: T. Brett Reece, MD. Division of Cardiothoracic Surgery, Department of Surgery, University of Colorado School of Medicine, \\ Anschutz Medical Campus, 12631 E. 17th Avenue, C-310, Aurora, CO 80045, USA. Email: brett.reece@cuanschutz.edu.
}

\begin{abstract}
Acute type A aortic dissection (ATAD) carries extremely high morbidity and mortality with a significantly increased risk of cerebrovascular injury following repair compared to elective arch operations. Advancement in operative and neuroprotective strategies have led to a temporal improvement in outcomes, but the incidence of cerebrovascular injury remains high and warrants continued innovation. In the setting of ATAD, stroke can occur due to malperfusion resulting from true lumen compression and compromised cerebral inflow, malperfusion from cardiogenic shock, or embolization. Therefore, stroke mitigation strategies must address vascular obstructions, hemodynamic compromise, and reduce risk of embolization. This article reviews contemporary strategies for cerebral protection in ATAD including preoperative patient evaluation, perfusion and temperature management, extent of aortic repair, utility of cerebral oximetry and neuromonitoring, and pharmacological adjuncts. In summary, patients should be evaluated preoperatively with contrast imaging to fully characterize their dissection to facilitate well-informed operative planning. Cannulation for cardiopulmonary bypass (CPB) can be safely achieved through multiple routes, but should consider patient pathology and the intended approach to cerebral perfusion. Central cannulation is feasible in most patients and allows for the most expeditious initiation of CPB. Although both deep hypothermic circulatory arrest (HCA) with retrograde cerebral perfusion (RCP) and moderate HCA with antegrade cerebral perfusion (ACP) have been shown to provide adequate cerebral protection, targeting moderate hypothermic temperatures reduces $\mathrm{CPB}$ times. The combination of a brief period of RCP followed by ACP during moderate HCA allows for the potential flushing of emboli from the cerebral arterial system with the superior metabolic protection of antegrade perfusion. The proximal and distal extent of arch repair should be individually tailored to patient pathology and surgeon experience, making sure to completely resect disease to prevent acute neurological complications. Cerebral oximetry and neuromonitoring are useful adjuncts, providing real-time information about the adequacy of neuroprotection. Finally, co-management by anesthesiologists and perfusionists with administration of neuroprotective agents, appropriate $\mathrm{pH}$ management, and maintenance of normal metabolic hemostasis and hemodynamic stability will further minimize cerebrovascular complications. Optimal cerebral protection requires a comprehensive, interdisciplinary approach and implementation of multimodal strategies.
\end{abstract}

Keywords: Cerebral protection; neuroprotection; acute type A aortic dissection (ATAD); aortic arch surgery

Received: 16 March 2020; Accepted: 01 June 2020; Published: 20 October 2021.

doi: 10.21037 /jovs-20-82

View this article at: http://dx.doi.org/10.21037/jovs-20-82 


\section{Introduction}

Acute type A aortic dissection (ATAD) carries extremely high morbidity and mortality. Retrospective studies have reported operative mortality ranging from $5-23 \%$ (1). Data from the International Registry of Acute Aortic Dissection (IRAD) demonstrated a temporal improvement in operative mortality from $25 \%$ in the late 1990 s to $18 \%$ in the early 2010s, which was accompanied by an increased rate of operative intervention for patients presenting with ATAD, suggesting operative approach has improved over time (2). Despite these promising improvements, adverse events remain high.

ATAD patients are at significantly increased risk of cerebrovascular injury compared to those undergoing elective arch operations for other pathologies (3). The incidence of stroke following ATAD repair ranges from $0.9-10.2 \%$ for transient neurologic deficits and $2.9-18.9 \%$ for permanent neurologic deficits, and varies depending on patient risk factors, cerebral protection strategy, and extent of arch surgery (1). These patients also experience increased mortality, healthcare costs, and diminished quality of life $(4,5)$.

Stroke can present with a variety of symptoms based on a spectrum of pathology. Classically, the most common stroke arises from carotid involvement with true lumen compression and compromised cerebral perfusion. Other complicating factors such as coronary involvement and pericardial effusion can lead to cardiogenic shock and brain malperfusion. While the malperfusion type syndromes are more commonly discussed, embolism remains a significant cause of stroke $(3,6-8)$. For many years, the group from Penn pushed for a no clamp technique $(9,10)$ given evidence that aortic cross-clamping increased permanent neurological deficits (11). Though other large series found it to be safe $(12,13)$, patients with hematoma or atheroma can undoubtedly embolize with proximal aortic manipulation. Finally, any intimal defect can serve as a nidus for embolization. This is more common in the chronic patients but can cause issues as with any anatomic source for flow anomalies. Stroke can essentially be caused by both vascular obstruction and embolism.

The goals of ATAD repair are to: (I) reestablish true lumen flow; (II) replace the diseased ascending aorta and/or aortic arch under hypothermic circulatory arrest (HCA) to achieve a sufficient resection of entry tears; (III) obliterate false lumen flow; (IV) adequately protect the brain and distal organs using a combination of perfusion techniques and hypothermia. This article reviews contemporary strategies for cerebral protection in ATAD including preoperative patient evaluation, perfusion and temperature management, extent of aortic repair, utility of cerebral oximetry and neuromonitoring, and pharmacological adjuncts.

\section{Presentation and preoperative evaluation}

A majority of ATAD patients (79\%) present with sudden onset severe chest pain, though the classic tearing or ripping sensation is not commonly described (2). The diagnostic gold standard for ATAD is computed tomography angiogram (CTA) of the chest. Ideally the CTA would also include the neck, abdomen, and pelvis to fully evaluate distal extent of dissection in the cranial vessels and descending aorta. CTA imaging is not only diagnostic, but also prognostic and facilitates operative planning. Based on CTA findings, the surgeon can often determine the extent of arch operation indicated and select cannulation site for cardiopulmonary bypass (CPB) and cerebral perfusion. The location and extension of dissection, arch vessel involvement or occlusion, and presence of intramural hematoma are important variables that guide these decisions (Figure 1).

Initial evaluation should also include physical exam to assess for clinical signs of stroke or malperfusion, and blood tests that may indicate metabolic derangements suggestive of malperfusion (lactate, $\mathrm{pH}$, base deficit, creatinine, liver function tests). Sultan et al. found that $15.1 \%$ of IRAD patients presented with cerebral malperfusion, placing them at significantly higher risk of postoperative stroke $(17.5 \%$ vs. $7.2 \%, \mathrm{P}=0.012$ ) and mortality (14). Even so, cerebral malperfusion should not preclude timely operative intervention as in-hospital survival ranges from $75-78 \%(14,15)$.

Most risk factors for postoperative stroke, including atherothrombotic disease and cerebral malperfusion at presentation, are non-modifiable (16-18). However, operative management can mitigate risk by careful identification of atheromatous disease and dissection anatomy to avoid propagation of emboli and reestablish cerebral perfusion if compromised. Further risk reduction can be achieved by minimizing CPB time, which is a known risk factor for postoperative stroke (4).

\section{Perfusion and temperature management}

\section{Arterial cannulation for $C P B$}

There are multiple arterial cannulation options available for initiating CPB: axillary, innominate, ascending aortic, 

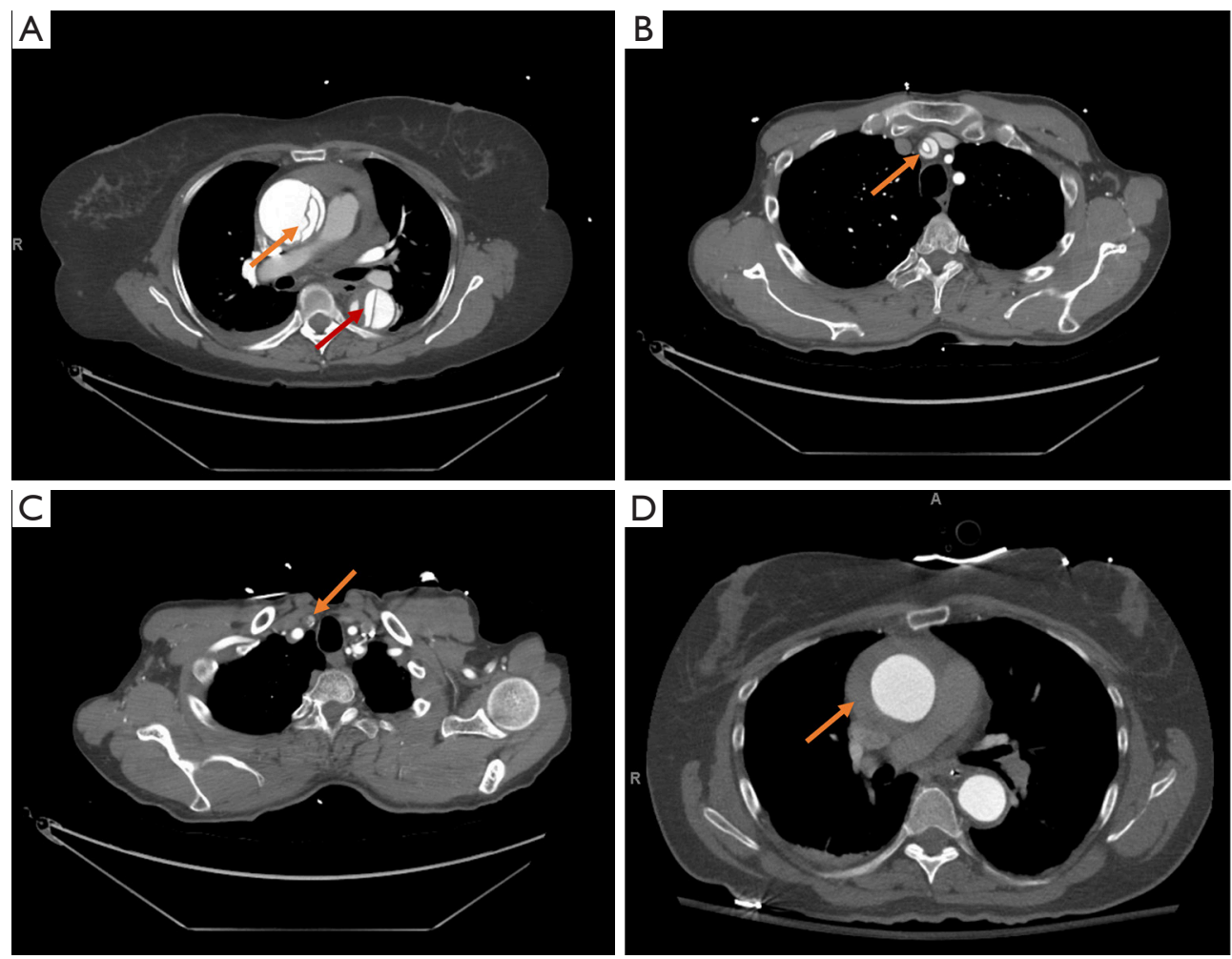

Figure 1 Radiological findings in patients presenting with acute type A dissection. (A) Axial computed tomography image in an acute type A dissection patient showing complex ascending aortic entry tear and dissection (orange arrow); the dissection extends into the descending aorta with true lumen compression (red arrow). (B) Axial computed tomography image in an acute type A dissection patient showing circumferential dissection of the innominate artery (orange arrow). (C) Axial computed tomography image in an acute type A dissection patient showing right common carotid artery malperfusion due to oblideration of the true lumen (orange arrow). (D) Axial computed tomography image in a patient with circumferential intramural hematoma (orange arrow).

femoral, and carotid. Although no single approach has been definitively shown to be superior in terms of neurological outcomes, each has advantages and disadvantages that should be considered when tailoring to individual patients (19).

Axillary artery cannulation is a safe approach (Figure 2A), as the artery is easy to access and control through an incision in the deltopectoral groove, and establishes arterial inflow prior to sternotomy (19-22). This can be achieved by sewing a side-graft to the vessel or through direct cannulation using the Seldinger technique, though direct cannulation can lead to upper extremity hypoperfusion or ischemia on the ipsilateral side (22). Antegrade cerebral perfusion (ACP) during HCA arrest can also be maintained through right axillary cannulation, while left axillary cannulation would require an alternative cannulation site for ACP. Right axillary cannulation may also flush aortic or innominate emboli retrograde, providing a hypothetical neuroprotective advantage. A disadvantage of axillary cannulation is potential limitations in achievable flow through the bypass circuit due to the small caliber of the vessel or if the vessel is dissected proximally. Moreover, repairing dissection in the vessel may be difficult if involved.

Innominate artery cannulation (Figure $2 B$ ) does not require an additional incision compared to axillary cannulation, which saves time, but may be disadvantageous in patients presenting with hemodynamic instability who might require more expeditious initiation of bypass $(19,22)$. The larger caliber of the vessels leads to improved flows, which can reduce cooling times. Innominate cannulation for $\mathrm{CPB}$ can be established by sewing a side-graft to the artery and ACP can be maintained with the same arterial cannula during HCA. During cross-clamping, regional cerebral oximetry should be monitored using near infrared 
A

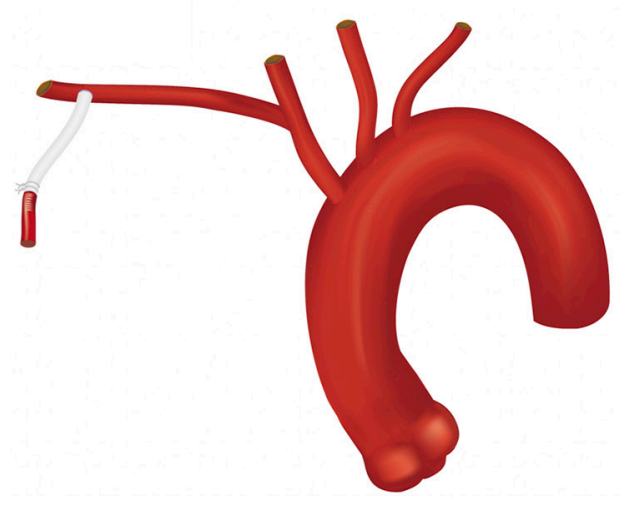

C

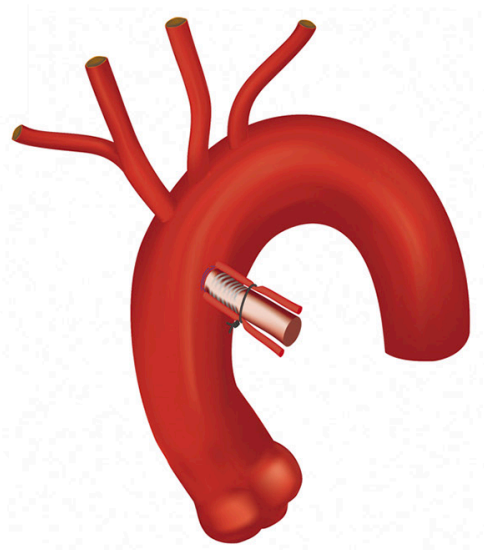

B

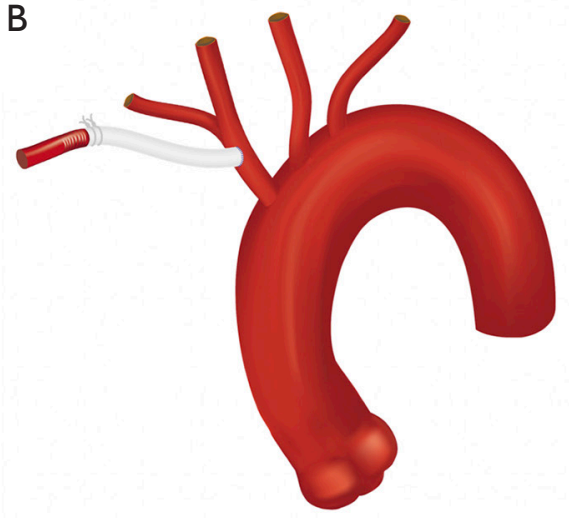

D

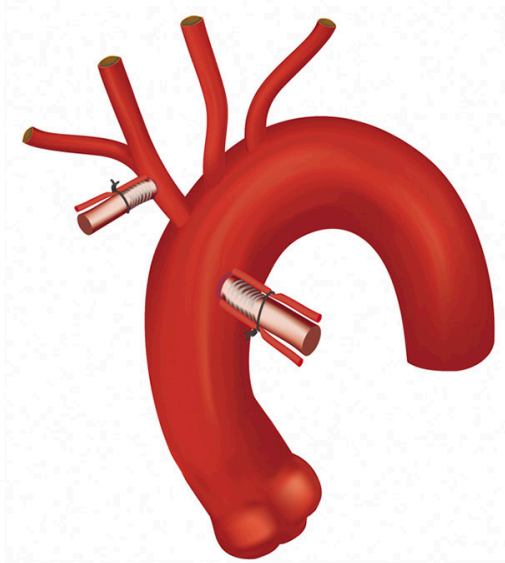

Figure 2 Arterial cannulation strategies to establish cardiopulmonary bypass. (A) Right axillary artery cannulation using a tube graft sewn end-to-side. (B) Innominate artery cannulation using a tube graft sewn end-to-side. (C) The ascending aorta is cannulated for initiation of bypass; this can be safely achieved even in dissected patients using the Seldinger technique. (D) In patients undergoing hemiarch repair, after the ascending aorta is cannulated for initiation of cardiopulmonary bypass and cooling, the innominate artery is cannulated using the Seldinger technique for administration of antegrade cerebral perfusion.

spectroscopy (NIRS) to ensure the right hemisphere is being adequately perfused. If the vessel is dissected however, it may not be the optimal choice for cannulation (19).

Common carotid artery cannulation is an available option on either side and is a good, expedient option to addressing cerebral malperfusion at presentation (23). The vessel can be accessed through a vertical incision overlying the sternocleidomastoid one to two finger breadths above the clavicle with a side-graft sewn to the vessel $(19,22,23)$. NIRS should be used to monitor for concerns of inadequate cerebral perfusion. Advantages to this approach are easy access, establishment of arterial inflow prior to sternotomy, and the ability to perfuse the brain during HCA through the same cannula. Potential downsides to carotid cannulation are lower flow compared to more central cannulation, inability to perfuse the body if the vessel is dissected proximally, and excessive flow pressure directed to the brain if the vessel is obstructed proximally $(19,22,23)$.

Femoral artery cannulation is a safe alternative especially in hemodynamically unstable patients, as they can be placed on bypass prior to sternotomy. The vessel may be accessed by open cutdown or ultrasound guided percutaneous cannulation of either groin (22). Separate cannulation will be necessary for cerebral perfusion during HCA, however.

At our institution, the preferred cannulation approach has evolved over time and our current preference is ascending aortic cannulation (Figure 2C) for expeditious initiation of $\mathrm{CPB}$ and cooling (24). We use preoperative CTA, epi-aortic ultrasound, and transesophageal echocardiography (TEE) to guide technique. If the anterior ascending aorta does 
not have significant pathology (dissection, calcification, or atherothrombotic disease), then the traditional method for aortic cannulation is employed. On the other hand, if the anterior aorta is dissected we cannulate using the Seldinger technique over a wire with TEE confirmation of true lumen access $(19,22,25,26)$. Excessive anterior aortic calcification or thrombus within the ascending true lumen can be prohibitive to ascending cannulation due to risk of embolization, in which case axillary, innominate, or carotid cannulation may be preferred. Nevertheless, aortic cannulation has been feasible in a majority of patients and resulted in significant reductions in operative times compared to axillary or innominate cannulation given that no additional incision is necessary and CPB can be initiated quickly once sternotomy is performed without the need for constructing an anastomosis prior. In these patients, the route of cerebral perfusion is established separately during cooling.

No cannulation strategy is optimal for all patients. We retain the abovementioned cannulation strategies in our armamentarium should one be specifically advantageous based on patient presentation or anatomy. The surgeon should be wary that based on dissection anatomy, certain cannulation modalities can worsen or induce cerebral malperfusion. Cerebral oximetry and neuromonitoring should be closely observed to recognize this possibility. In the setting of cerebral malperfusion due to common carotid dissection with true lumen obliteration, patients may require multiple routes of perfusion to reestablish cerebral blood flow by direct carotid cutdown and to initiate CPB (19).

\section{Target nadir temperature and cerebral perfusion}

The concept hypothermia for cerebral protection and end organ protection during HCA was established by Dr. Griepp (7) and has become a principal tenet of aortic arch repair. Decreased temperatures reduce cellular metabolic demand, therefore reducing the risk of ischemic injury while organs are not being perfused.

The optimal target temperature for HCA (mild $>28^{\circ} \mathrm{C}$, moderate $20-28{ }^{\circ} \mathrm{C}$, or deep $<20^{\circ} \mathrm{C}$ hypothermia) remains open for debate, as adequate lower body protection has been demonstrated even with the warmer temperature ranges (27-35). Therefore, the target temperature range is largely determined by the adjunctive cerebral perfusion strategy. Many surgeons still perform the distal anastomosis under deep HCA with no cerebral perfusion, however there is a growing body of evidence that deep HCA with no perfusion is inferior to HCA with some form of cerebral perfusionantegrade or retrograde-in terms of neurological outcomes (28,36-39). Even though hypothermia reduces metabolic demand, it does not achieve complete cellular quiescence, so cells continue to require oxygenation and nutrition (19). Most aortic centers now prefer moderate HCA with ACP or deep HCA with retrograde cerebral perfusion (RCP), both demonstrating equivocal outcomes neurologically and overall $(27,35-38,40-43)$. The basis for pairing RCP with colder temperatures is the commonly accepted perception that retrograde flow does not provide the same nutritional support for the brain as ACP, which has been shown in animal models of HCA $(44,45)$.

Recently, the Canadian Thoracic Aortic Collaborative published a large multicenter study evaluating outcomes following different cerebral perfusion and temperature management strategies; 2,520 patients were included. The study found that ACP (compared to no cerebral perfusion) and temperatures $\geq 24{ }^{\circ} \mathrm{C}$ were protective against stroke, mortality, and other morbidity. There were no statistically significant differences in outcomes comparing ACP and RCP, though the number of RCP patients was small. These findings lead the authors to conclude that surgeons should provide cerebral perfusion during circulatory arrest and adopt warmer temperature targets. However, the data did not favor one cerebral perfusion strategy over another (46).

Over time, at our institution we have been targeting warmer temperatures. Our currently preferred target core temperature during HCA is moderate $\left(26-28{ }^{\circ} \mathrm{C}\right)$ for two main reasons: its safety with ACP is robustly validated and warmer temperatures mean faster cooling and rewarming, leading to shorter CPB time and thus theoretically further reducing stroke risk.

Regarding cannulation for ACP, our preference has been providing unilateral ACP. Unilateral perfusion has been shown to effectively protect the brain under hypothermia in a majority of patients through the circle of Willis and extracranial collaterals, particularly for HCA times $<40$ minutes (47-50). Early in our experience we primarily cannulated patients via the right axillary with a sidegraft, followed by a transition to sewing a side-graft to the innominate. As we subsequently adopted aortic cannulation for CPB, the cannulation choice for ACP became much more flexible and is now determined by the extent of arch operation.

For hemiarch repair we directly cannulate the innominate with a $14 \mathrm{~F}$ cannula using the Seldinger technique, which 


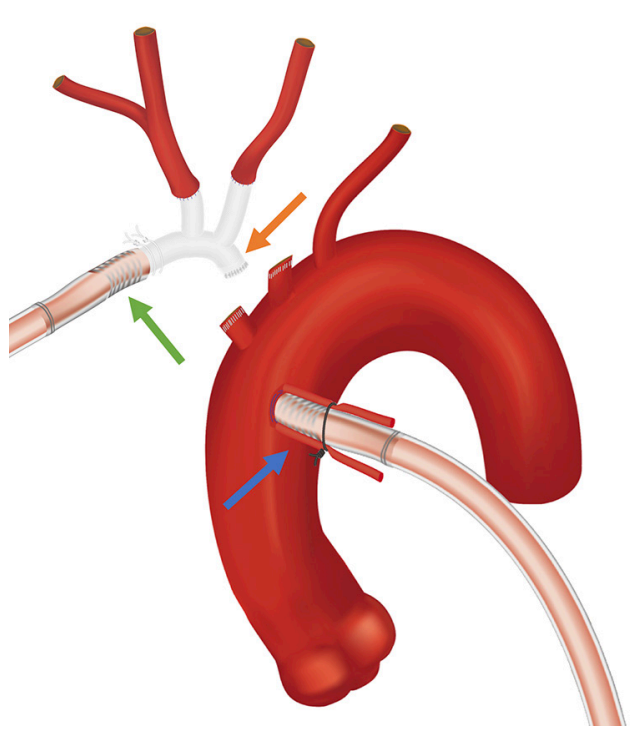

Figure 3 This figure shows the "branch-first" technique which we use for arch vessel management in total arch replacement. Arterial inflow for cardiopulmonary bypass is achieved with ascending aortic cannulation (blue arrow). The innominate and left common carotid arteries are then sequentially anastomosed to a trifurcated graft with a perfusion side-arm that is connected to a second arterial line of the bypass circuit (green arrow), which allows for continuous cerebral perfusion during the process; unilateral perfusion is maintained while each branch vessel is being anastomosed, with bilateral perfusion once completed. Once the distal arch graft anastomosis is complete and the body is being reperfused, the staple line of the proximal large branch of the trifurcated graft (orange arrow) is cut and anastomosed to the proximal branch of the arch graft as shown in Figure 4.

saves a significant amount of time compared to sewing a side-graft (Figure 2D). Alternatively, when performing total arch replacement (TAR), we now employ a "branch first" technique (Figure 3) whereby the innominate and left common carotid arteries are sequentially anastomosed to a trifurcated graft as described by Spielvogel et al. $(50,51)$. Our approach is unique in that we connect one of the trifurcated graft branches to a separate arterial line, allowing for continuous cerebral perfusion during the process; unilateral perfusion is maintained while each branch vessel is being anastomosed, with bilateral perfusion once completed. NIRS is carefully monitored during innominate and left common carotid artery cross-clamping. Thus, once the body has been sufficiently cooled, only the distal anastomosis remains to be constructed during HCA for TAR. Importantly, for both hemiarch and TAR, we avoid aortic cross-clamping prior to HCA, to minimize risk of embolization (11).

Despite improvements in our operative technique, we have noted that patients can develop cerebral infarcts bilaterally regardless of extent of arch repair and even when risk factors for embolic stroke were not immediately apparent (Figure 5). These observations are consistent with the findings of a recent prospective study published by Leshnower et al, which compared neurological outcomes between patients undergoing DHCA with RCP vs. MHCA with ACP (29). Although there was no clinical difference in outcomes between the groups, $100 \%$ of ACP patients had MRI lesions consistent with embolic stroke compared with $45 \%$ of RCP patients $(\mathrm{P}=0.01)$ and the mean number of MRI lesions was significantly higher in ACP patients: $4 \pm 3.5$ vs. $1.2 \pm 2.1, \mathrm{P}=0.01$ (29). Interestingly, strokes were most commonly found in the bilateral frontal lobes, suggesting that emboli must be getting propagated throughout CPB from aortic and arch vessel manipulation, not just during the period of ACP. Similar anecdotal observations in our own patients prompted us to combine the metabolic benefits of ACP with the potential benefit of RCP to flush out embolic debris in a strategy called "Shaggy Aorta" protocol. This was inspired by the work of Dr. Yokawa et al. (52) who found that patients with aortic atheroma were at increased risk of spinal cord injury during thoracoabdominal aortic repair. Likewise, in arch operations, whether presenting with atheroma or false lumen hematoma, we felt that these patients were at particularly high risk for cerebrovascular complications; and while patients with ATAD are far less likely than chronic dissection patients to have false lumen thrombus, embolic stroke remains a significant risk.

\section{"Shaggy Aorta" protocol}

The principle of "Shaggy Aorta" protocol is to administer a brief period of RCP at the beginning of HCA to washout possible embolic debris followed by ACP for the remainder of HCA. We initially applied this strategy in urgent/ emergent and high-risk patients but have most recently begun to employ it in all patients undergoing open aortic arch operations. The ability to quickly and easily switch the route of arterial inflow has been greatly facilitated by routinely splitting our $\mathrm{CPB}$ arterial line twice, providing three possible routes of inflow. One line is brought along the patient's left and the remaining two along the patient's right. The line along the patient's left is connected to the aortic cannula for the initiation of bypass and is later 

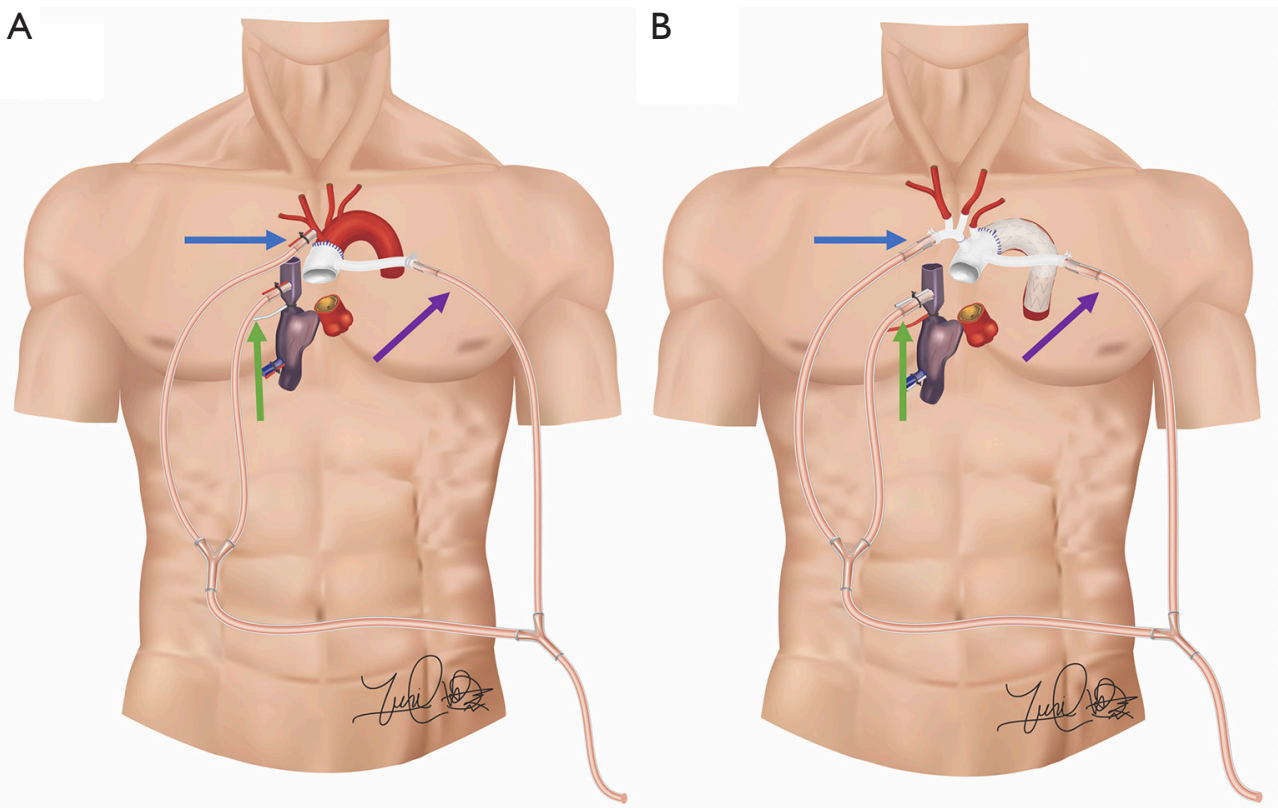

Figure 4 "Shaggy Aorta" protocol arterial management in open aortic arch repair. (A) This figure demonstrates arterial management using the "Shaggy Aorta" protocol in hemiarch replacement. Patients are cannulated centrally for initiation of bypass. At the start of hypothermic circulatory arrest, a brief period of retrograde cerebral perfusion is administered through the superior vena cava (green arrow) followed by antegrade cerebral perfusion through an innominate artery cannula (blue arrow). Once the distal anastomosis is complete, the patient is reperfused through a side-arm on the hemiarch graft (purple arrow). (B) This figure shows arterial management using the "Shaggy Aorta" protocol in total arch replacement. Patients are cannulated centrally for initiation of bypass. At the start of hypothermic circulatory arrest, a brief period of retrograde cerebral perfusion is administered through the superior vena cava (green arrow) followed by antegrade cerebral perfusion through a trifurcated graft sewn to the innominate and left common carotid arteries (blue arrow). Once the distal anastomosis is complete, the patient is reperfused through a side-arm on the arch graft (purple arrow).

connected to the perfusion limb of the hemiarch or arch graft to re-perfuse the body at the termination of HCA. The two inflow lines along the patient's right are used for RCP and ACP (Figure 4). "Shaggy Aorta" protocol is described in Video 1.

In our early experience, no patients developed stroke after undergoing open aortic arch operations utilizing the "Shaggy Aorta" protocol. Table 1 summarizes pathology and operative characteristics for the first 29 patients in whom this strategy was applied. Urgent or emergent procedures were performed in $44.8 \%(13 / 29)$ of patients, 5 of whom $(17.2 \%)$ presented with ATAD. The remaining 16 cases $(55.2 \%)$ were elective arch operations. All patients underwent median sternotomy. Arterial cannulation for $\mathrm{CPB}$ varied among patients depending on anatomy and pathology, but a majority were cannulated centrally. After cooling to moderate hypothermia $\left(26-28^{\circ} \mathrm{C}\right)$, HCA was initiated for reconstruction of the distal anastomosis. For 22 patients, a 3-minute period of RCP was administered through the SVC at a rate of $1.5-2 \mathrm{~L} / \mathrm{min}$ with a goal central venous pressure of 20-30 at the start of HCA followed by ACP-mode of cannulation is specified in Table 1 . In the remaining 7 patients, only RCP was administered during circulatory arrest as the HCA period was anticipated to be short. Although, as discussed previously, RCP has been exclusively accompanied by colder target nadir temperatures, use of moderate hypothermia in our patients appears safe. Our early experience utilizing a strategy of RCP for the washout of potential arterial thromboembolic debris as an adjunct in aortic arch repair with moderate HCA shows promising results in the improvement of cerebral protection with no cerebrovascular complications.

\section{Choice of reconstruction}

\section{Arch extent and proximal repair}

Widely debated and without clear resolution are the 
questions of how extensive the arch replacement should be and how to best address the root proximally. Regarding arch extent, hemiarch remains the standard of care and is an appropriate operation in a majority of patients $(53,54)$.

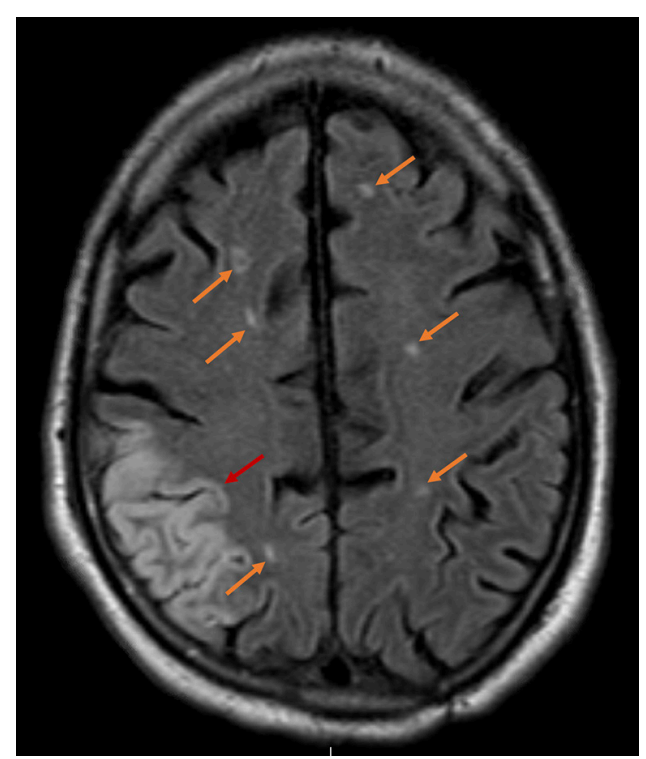

Figure 5 Axial magnetic resonance imaging cut showing bilateral embolic infarcts (orange arrows) and one large ischemic right middle cerebral artery territory infarct in a male patient diagnosed with stroke postoperatively after undergoing total arch replacement with frozen elephant trunk.

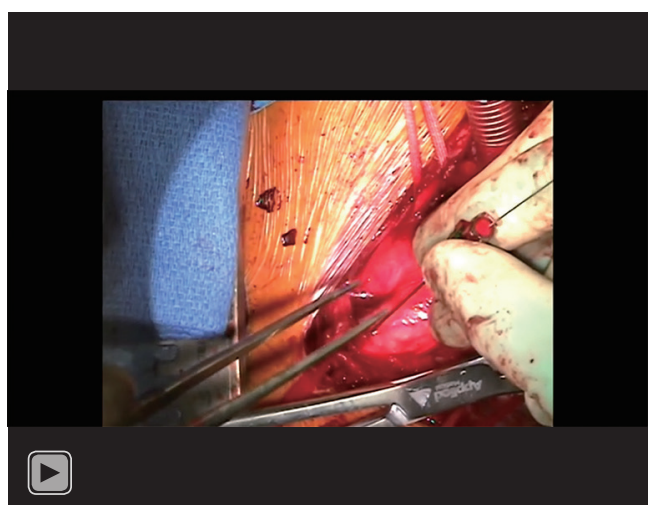

Video 1 This video summarizes the development of the "Shaggy Aorta" protocol including the observation of persistent bilateral embolic infarcts in patients undergoing open aortic arch operations despite advancements in neuroprotective strategies, the technical details of the protocol in both hemiarch and total arch patients, and early outcomes of this strategy.
The goals of hemiarch ATAD repair are resection of the primary entry if present in the ascending aorta or the lesser curvature of the arch, exclusion of the false lumen in the construction of the distal anastomosis, and ensuring the

Table 1 Patient and operative characteristics for 29 "Shaggy Aorta" Protocol patients

\begin{tabular}{|c|c|}
\hline Patient characteristics & $\mathrm{N}(\%)^{*}$ \\
\hline Age & $58.0 \pm 12.7$ \\
\hline Female gender & $10(34.5)$ \\
\hline BMI $\left(\mathrm{kg} / \mathrm{m}^{2}\right)$ & $28.6 \pm 5.8$ \\
\hline Hypertension & $18(62.1)$ \\
\hline Hyperlipidemia & $9(31.0)$ \\
\hline Coronary artery disease & $5(17.2)$ \\
\hline Chronic kidney disease & $11(37.9)$ \\
\hline Stroke prior to presentation & $4(13.8)$ \\
\hline Related to pathology? & $3(10.3)$ \\
\hline \multicolumn{2}{|l|}{ Presentation/pathology } \\
\hline Type A dissection & $10(34.5)$ \\
\hline Acute presentation & $5(17.2)$ \\
\hline Arch vessel involvement & $5(17.2)$ \\
\hline Brain malperfusion & $2(6.9)$ \\
\hline Root/ascending aneurysm & $24(82.8)$ \\
\hline Porcelain ascending aorta & $1(3.4)$ \\
\hline Ascending aortic atheroma & $1(3.4)$ \\
\hline Urgent/emergent & $13(44.8)$ \\
\hline Redo sternotomy & $10(34.5)$ \\
\hline \multicolumn{2}{|l|}{ Mode of arterial cannulation for bypass } \\
\hline Ascending aorta & $25(96.6)$ \\
\hline Innominate artery & $2(6.9)$ \\
\hline Right axillary artery & $1(3.4)$ \\
\hline Femoral artery & $1(3.4)$ \\
\hline \multicolumn{2}{|l|}{ Mode of SACP cannulation } \\
\hline Innominate & $12(41.4)$ \\
\hline "Branch First" (innominate and LCCA) & $9(31.0)$ \\
\hline Right axillary artery & $1(3.4)$ \\
\hline Retrograde only & $7(24.1)$ \\
\hline
\end{tabular}

Table 1 (Continued) 
Table 1 (Continued)

\begin{tabular}{lc}
\hline Patient characteristics & $\mathrm{N}(\%)^{\star}$ \\
\hline Operative characteristics & \\
CPB time (min) & $172.7 \pm 55.7$ \\
SACP time (min) & $12.3 \pm 6.8$ \\
Nadir bladder temp $(\boldsymbol{C J})$ & $26.7 \pm 1.4$ \\
Hemiarch repair & $17(58.6)$ \\
Total arch repair & $12(41.4)$ \\
Frozen elephant trunk & $7(24.1)$ \\
Aortic root replacement & $14(48.3)$ \\
Isolated aortic valve replacement & $7(24.1)$ \\
CABG & $3(10.3)$
\end{tabular}

*, categorical variables are expressed as frequency and column percent and continuous variables are expressed as mean \pm standard deviation. BMI, body mass index; SACP, selective antegrade cerebral perfusion; LCCA, left common carotid artery; $\mathrm{CPB}$, cardiopulmonary bypass; CABG, coronary artery bypass grafting.

patient survives the acute phase of their disease. Especially when patients are critically ill/unstable or at low volume centers, a more limited repair is likely the best option in a majority of patients $(55,56)$. However, for tears located along the greater curvature, significantly dissected arch vessels, or if patients present with cerebral malperfusion, there is evidence that TAR with aggressive resection of diseased branch vessels yields improved neurological outcomes in the acute phase (57). Late neurological outcomes may also be impacted if arch vessel disease is not addressed at presentation. Anecdotally, at our institution we have encountered three patients who presented with stroke or transient ischemic attacks due to showering of thromboemboli from residual arch or arch vessel dissection after hemiarch repair years prior. These patients required aortic reintervention with TAR to address their remaining disease.

Proponents of a pathology-directed expansion of the "tear oriented" approach described by Westaby et al. (58) include circumferential arch or arch vessel dissection, arch aneurysm, and descending dissection with true lumen compromise as indications for TAR $(53,57,59)$. Others argue that as outcomes of TAR performed at large volume institutions have improved and are similar to hemiarch outcomes (60), TAR should also be considered for the prevention of mid- or late-term aortic degeneration requiring reoperation $(54,61)$. Matalanis et al. propose more extensive arch repair in all ATAD patients using a standardized and simplified TAR technique that has yielded excellent early results $(61,62)$. Critics caution that outcomes from expert centers cannot be extrapolated to low-volume surgeons burdened with treating ATAD and therefore, TAR cannot reasonably become the standard of care (55,56,63-65). Furthermore aortic arch reintervention after hemiarch repair for ATAD remains low: $11.5 \%$ and $15 \%$ at 5 - and 10 -year respectively $(42,53,56)$. So, for the time being, the choice of arch extent remains a balancing act weighing operator skill and experience, acute patient pathology and stability, and the possibility of long-term aortic degeneration.

At our institution, we are trending toward favoring more extensive arch repair particularly in patients with complex arch, arch vessel, or descending aortic involvementsimilar to the considerations described by Trivedi et al. and Sultan et al. $(53,57)$. The decision for more extensive repair is driven by the need to resect and repair arch vessel disease, reestablish true lumen flow and distal perfusion in the setting of descending dissection and lower body malperfusion, and reduce the risk of late aortic arch reintervention. In patients with connective tissue disease or more extensive thoracoabdominal aortic disease, TAR with or without frozen elephant trunk (FET) facilitates future downstream aortic repair as well.

The "branch-first" technique of arch vessel management allows for dramatic reductions in circulatory arrest times, and while not strictly speaking necessary for cerebral protection, provides bilateral ACP during circulatory arrest. The Buffalo Trunk technique has also streamlined the construction of the distal anastomosis in TAR with FET resulting in further reductions in HCA time, which we have previously described (66). Shorter circulatory arrest times for TAR have made targeting warmer nadir temperatures safe, as we have been able to limit end organ ischemia.

Our hard indication for FET placement is ongoing lower body malperfusion. FET can also lay the groundwork for later endovascular extension of the repair in the setting of descending aortic pathology that will likely require future operative intervention. Though we used hemiarch with antegrade stent early in our experience and this remains the preferred strategy at certain centers, we now perform TAR if stent is needed using the Buffalo Trunk technique. It should be noted that for FET reconstruction we insert a guide-wire into the aortic arch from the groin verifying it is in the true lumen along its entire course using intravascular 
ultrasound; the stent graft device is placed over the wire during HCA, thereby ensuring the stent is deployed in the true lumen both proximally and distally.

The debate about the optimal approach to addressing the root in ATAD is analogous to the debate about arch extent. The conservative approach (root repair with reapproximation of the aortic wall, valve resuspension, and reconstruction of the sinotubular junction) is the most expeditious and reproducible in inexperienced hands but is least durable in the long-term $(67,68)$. High volume centers have demonstrated that root replacement with prosthetic valve and valve sparing root replacement can safely be performed with excellent long-term outcomes (69-72). That being said, the most prudent approach is likely an individualized one that takes into account surgeon expertise, patient anatomy and pathology (presence of root entry tear, extent of root dissection, extent of valve disruption, root dilation, connective tissue disorder), and patient stability (73-76).

In this regard, at our institution we are also trending toward a more aggressive strategy and favor replacement over repair if there is any question of root integrity, since these are safely performed in our hands. Our reasons for this are similar to more aggressive arch repair-residual root dissection can be a nidus for embolic complications and is likely to degenerate over time requiring reoperation.

\section{Cerebral oximetry and neuromonitoring}

For optimized cerebral protection, we employ a comprehensive neuro-cerebral monitoring strategy with regional cerebral oximetry using NIRS and neurophysiologic intraoperative monitoring (NIOM), which consists of electroencephalography (EEG), somatosensory evoked potentials (SSEPs), and motor evoked potentials (MEPs). These modalities are often used to guide cerebral circulatory management (77) and have been shown anecdotally to improve outcomes in cardiac surgery (78-80). Abnormalities in both cerebral oximetry and NIOM can indicate inadequate perfusion due to mispositioned cannula or arterial obstruction. EEG is also used to ensure cerebral electrical silence during HCA (Figure 6). At institutions using deep HCA, patients are cooled until electrical silence is attained. Since moderate hypothermic temperatures alone are not sufficient to induce electrical silence, once the target temperature is reached in our patients, propofol is administered to fully achieve EEG silence. Furthermore, NIOM can aid in the early detection of stroke (22). The utility of NIOM in the detection/prevention of spinal cord injury is well demonstrated and broadly accepted (81), but its value in the early identification of stroke has been unclear.

Recently we demonstrated that NIOM has a high sensitivity and specificity for the early detection of stroke (82). NIOM can detect both embolic strokes and neurologic injury related to hypoperfusion. Embolic strokes are likely to result in more subtle NIOM changes - for example, isolated, unilateral changes in SSEPs or MEPs, if sensory or motor pathways are involved. Alternatively, injuries that result from inadequate brain protection or hemispheric hypoperfusion would likely be accompanied by decreased regional cerebral oxygen saturation and global EEG slowing, in addition to SSEP and MEP changes. Intraoperative identification of deficits can lead to operative interventions such as correction of perfusion issues or administration of RCP if embolism is suspected to potentially reverse deficits. Early detection can also prompt earlier cerebral imaging and possible catheter-based interventions.

\section{Pharmacology}

Many pharmacologic agents have been evaluated for cerebral protection in cardiac and aortic surgery. Proposed agents function through a variety of mechanisms and include anti-inflammatories, antioxidants, anti-apoptotic agents, drugs that reduce neuronal excitotoxicity, drugs that mimic ischemic preconditioning, medications that reduce metabolic demand, and osmotic agents to reduce tissue swelling. Table 2 lists multiple agents that were used historically, are currently used clinically, or have shown promising results in animal experiments (3,77,83-88). Many drugs have theoretical or anecdotal clinical benefits, or demonstrated promising results in experimental models, however, few have been robustly validated by clinical trials. Regarding historical agents, the days of heavy hitters (barbiturates or other coma-inducing agents) are gone, since our goal is to wake patients up as quickly as possible postoperatively to obtain a neurological exam.

We have previously described our institutional approach to pharmacological cerebral protection $(77,83)$. In short, we administer $500 \mathrm{mg}$ of methylprednisolone upon induction, followed by $100 \mathrm{mg}$ of lidocaine and $2 \mathrm{~g}$ of magnesium immediately before circulatory arrest once the patient has reached the target nadir temperature $(77,83)$. Propofol is also bolused to achieve EEG silence prior to HCA. 


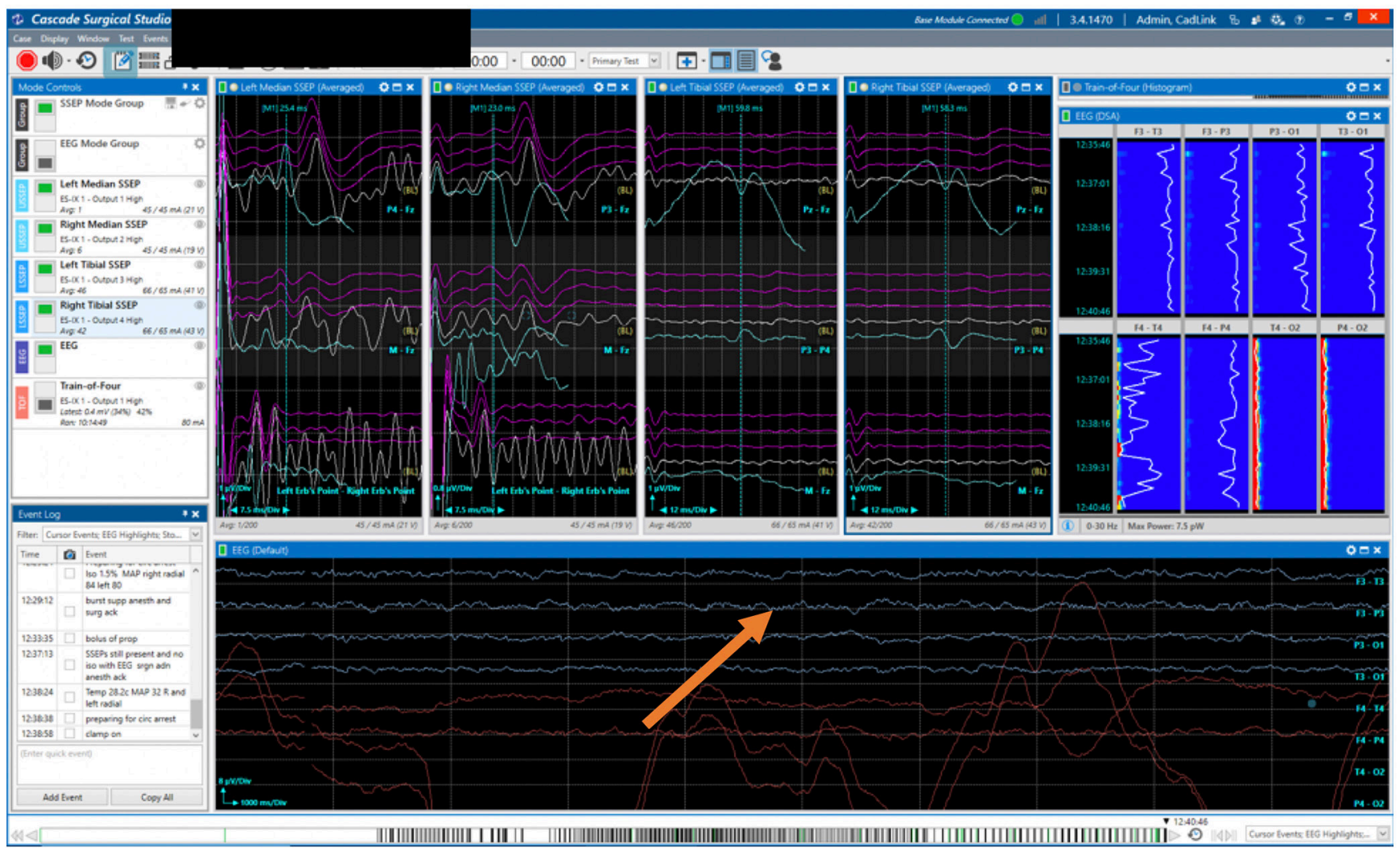

Figure 6 Image of neurophysiologic intraoperative monitoring during hemiarch replacement in a male. The orange arrow highlights that electrical silence has been achieved on electroencephalography prior to initiation of circulatory arrest.

\section{Additional considerations}

There is currently no consensus on which $\mathrm{pH}$ management strategy is optimal-alpha-stat, which measures $\mathrm{pH}$ at normothermia $\left(37^{\circ} \mathrm{C}\right) v s$. $\mathrm{pH}$-stat, which corrects $\mathrm{pH}$ to actual patient temperature $(77,86)$. Our perfusionists initially use alpha-stat at the beginning of $\mathrm{CPB}$ to correct any underlying metabolic derangements. During cooling and HCA, pH-stat is used to optimize cerebral blood flow (maintaining normal temperature-corrected $\mathrm{pCO}_{2}$ prevents cerebral vasoconstriction). Once rewarming, the perfusionists switch to alpha-stat and quickly blow off $\mathrm{CO}_{2}$ to promote normal cell homeostasis and potentially reduce cerebral edema and hyperthermia through relative vasoconstriction compared to $\mathrm{pH}$-stat (77).

During HCA our anesthesiologists topically cool the head, which may provide a more uniform cooling in patients with cerebral circulation abnormalities, though there is not strong evidence to support this widely used practice $(77,83)$. Lastly, we maintain a normal hematocrit above the transfusion threshold at $25-30 \%$ to provide adequate cerebral oxygenation and prevent vasoconstriction, especially during HCA (77).

\section{Summary}

Stroke following open aortic arch surgery is a devastating complication and patients presenting with ATAD are at significantly increased risk for cerebrovascular complications. Certainly, there are several non-modifiable risk factors for post-operative stroke, however contemporary perioperative cerebral protection strategies for cerebral protection have resulted in continued improvements in neurological outcomes following ATAD repair. Patients should be evaluated preoperatively with contrast imaging to fully characterize their dissection to facilitate well-informed operative planning. Central cannulation is feasible in most patients and allows for the most expeditious initiation of CPB. Moderate hypothermic temperatures provide adequate organ protection without unnecessarily prolonging 
Table 2 Pharmacologic agents for cerebral protection

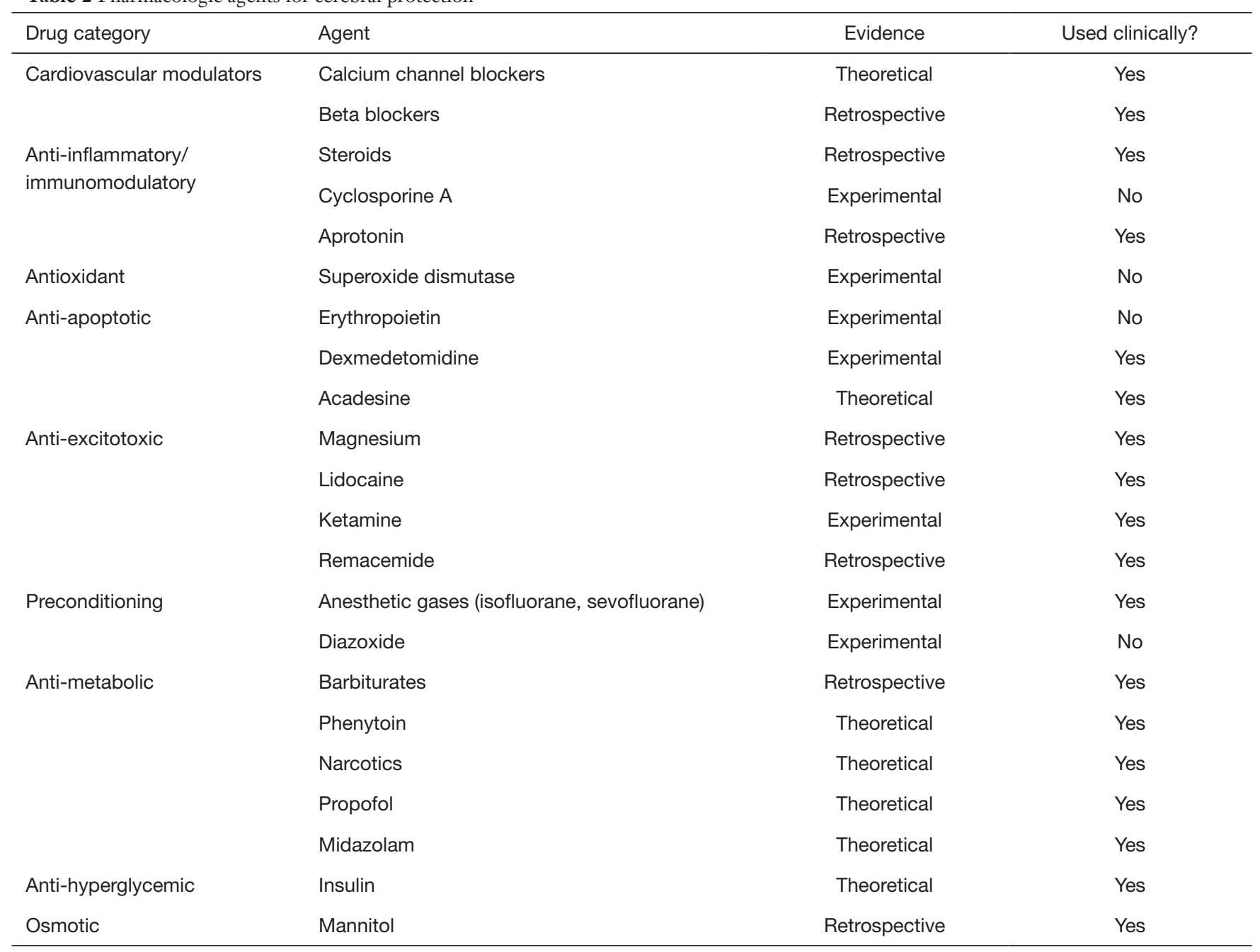

CPB. The combination of a brief period of RCP followed by ACP during HCA allows for the potential flushing of emboli from the cerebral arterial system with the superior metabolic protection of antegrade perfusion. The proximal and distal extent of arch repair should be individually tailored to patient pathology and surgeon experience, making sure to sufficiently resect disease to prevent acute neurological complications. Cerebral oximetry and NIOM are useful adjuncts in aortic surgery, providing real-time information about the adequacy of neuroprotection. Finally, co-management by anesthesiologists and perfusionists with administration of neuroprotective agents, appropriate $\mathrm{pH}$ management, and maintenance of normal metabolic hemostasis and hemodynamic stability will further minimize cerebrovascular complications. Optimal cerebral protection requires a comprehensive, interdisciplinary approach and implementation of multimodal strategies, which can achieve acceptable neurological outcomes in a very sick patient population.

\section{Acknowledgments}

Funding: This study was funded by the Division of Cardiothoracic Surgery at the University of Colorado School of Medicine.

\section{Footnote}

Provenance and Peer Review: This article was commissioned by the Guest Editors (Ibrahim Sultan and George Arnaoutakis) for the series "Advancement in the Surgical Treatment of Aortic Dissection" published in Fournal of 
Visualized Surgery. The article has undergone external peer review.

Conflicts of Interest: All authors have completed the ICMJE uniform disclosure form (available at https:// jovs.amegroups.com/article/view/10.21037/jovs-20-82/ coif). The series "Advancement in the Surgical Treatment of Aortic Dissection" was commissioned by the editorial office without any funding or sponsorship. MA reports non-financial support from W.L. Gore \& Associates, other from W.L. Gore \& Associates, other from LivaNova PLC, outside the submitted work. The authors have no other conflicts of interest to declare.

Ethical Statement: The authors are accountable for all aspects of the work in ensuring that questions related to the accuracy or integrity of any part of the work are appropriately investigated and resolved. Written informed consent was obtained from the patients for publication of this study and any accompanying images and videos. A copy of the written consent is available for review by the Editorin-Chief of this journal.

Open Access Statement: This is an Open Access article distributed in accordance with the Creative Commons Attribution-NonCommercial-NoDerivs 4.0 International License (CC BY-NC-ND 4.0), which permits the noncommercial replication and distribution of the article with the strict proviso that no changes or edits are made and the original work is properly cited (including links to both the formal publication through the relevant DOI and the license). See: https://creativecommons.org/licenses/by-nc-nd/4.0/.

\section{References}

1. Pacini D, Murana G, Di Marco L, et al. Cerebral perfusion issues in type A aortic dissection. J Vis Surg 2018;4:77.

2. Evangelista A, Isselbacher EM, Bossone E, et al. Insights from the international registry of acute aortic dissection: A 20-year experience of collaborative clinical research. Circulation 2018;137:1846-60.

3. Lindsay H, Srinivas C, Djaiani G. Neuroprotection during aortic surgery. Best Pract Res Clin Anaesthesiol 2016;30:283-303.

4. Ghoreishi M, Sundt TM, Cameron DE, et al. Factors associated with acute stroke after type A aortic dissection repair: An analysis of the Society of Thoracic Surgeons National Adult Cardiac Surgery Database. J Thorac
Cardiovasc Surg 2020;159:2143-2154.e3.

5. Bossone E, Corteville DC, Harris KM, et al. Stroke and outcomes in patients with acute type a aortic dissection. Circulation 2013;128:S175-9.

6. Hagl C, Ergin MA, Galla JD, et al. Neurologic outcome after ascending aorta-aortic arch operations: Effect of brain protection technique in high-risk patients. J Thorac Cardiovasc Surg 2001;121:1107-21.

7. Griepp RB, Di Luozzo G. Hypothermia for aortic surgery. J Thorac Cardiovasc Surg 2013;145:S56-8.

8. Newman MF, Mathew JP, Grocott HP, et al. Central nervous system injury associated with cardiac surgery. Lancet 2006;368:694-703.

9. Bavaria JE, Pochettino A, Brinster DR, et al. New paradigms and improved results for the surgical treatment of acute type A dissection. Ann Surg 2001;234:336-42.

10. Bavaria JE, Brinster DR, Gorman RC, et al. Advances in the treatment of acute type A dissection: An integrated approach. Ann Thorac Surg 2002;74:S1848-52; discussion S1857-63.

11. Comas GM, Leshnower BG, Halkos ME, et al. Acute type A dissection: Impact of antegrade cerebral perfusion under moderate hypothermia. Ann Thorac Surg 2013;96:2135-41.

12. Immer FF, Aydin NB, Lütolf M, et al. Does aortic crossclamping during the cooling phase affect the early clinical outcome of acute type A aortic dissection? J Thorac Cardiovasc Surg 2008;136:1536-40.

13. Nishida H, Tabata M, Fukui T, et al. A systematic approach to improve the outcomes of type A aortic dissection. J Thorac Cardiovasc Surg 2017;154:89-96.e1.

14. Sultan I, Bianco V, Patel HJ, et al. Surgery for type A aortic dissection in patients with cerebral malperfusion: Results from the International Registry of Acute Aortic Dissection. J Thorac Cardiovasc Surg 2019;S00225223(19)32762-X.

15. Chiu P, Rotto TJ, Goldstone AB, et al. Time-to-operation does not predict outcome in acute type A aortic dissection complicated by neurologic injury at presentation. J Thorac Cardiovasc Surg 2019;158:665-72.

16. Djaiani GN. Aortic arch atheroma: Stroke reduction in cardiac surgical patients. Semin Cardiothorac Vasc Anesth 2006;10:143-57.

17. Amarenco P. Atherosclerotic disease of the aortic arch as a risk factor for recurrent ischemic stroke. N Engl J Med 1996;334:1216-21.

18. Di Tullio MR, Russo C, Jin Z, et al. Aortic arch plaques and risk of recurrent stroke and death. Circulation 
2009;119:2376-82.

19. Rylski B, Urbanski PP, Siepe M, et al. Operative techniques in patients with type A dissection complicated by cerebral malperfusion. Eur J Cardiothorac Surg 2014;46:156-66.

20. Di Eusanio M, Pantaleo A, Petridis FD, et al. Impact of different cannulation strategies on in-hospital outcomes of aortic arch surgery: A propensity-score analysis. Ann Thorac Surg 2013;96:1656-63.

21. Svensson LG, Blackstone EH, Rajeswaran J, et al. Does the arterial cannulation site for circulatory arrest influence stroke risk? Ann Thorac Surg 2004;78:1274-84; discussion 1274-84.

22. Leshnower BG. Cannulation strategies, circulation management and neuroprotection for type A intramural hematoma: Tips and tricks. Ann Cardiothorac Surg 2019;8:561-6.

23. Urbanski PP, Wagner M. Perfusion and repair technique in acute aortic dissection with cerebral malperfusion and damage of the innominate artery. J Thorac Cardiovasc Surg 2012;144:982-4.

24. Reece TB, Tribble CG, Smith RL, et al. Central cannulation is safe in acute aortic dissection repair. J Thorac Cardiovasc Surg 2007;133:428-34.

25. Shimura S, Odagiri S, Furuya H, et al. Echocardiographyguided aortic cannulation by the Seldinger technique for type A dissection with cerebral malperfusion. J Thorac Cardiovasc Surg 2020;159:784-93.

26. Okita Y, Okada K, Omura A, et al. Total arch replacement using selective antegrade cerebral perfusion as the neuroprotection strategy. Ann Cardiothorac Surg 2013;2:169-74.

27. Keeling WB, Tian DH, Leshnower BG, et al. Safety of Moderate Hypothermia With Antegrade Cerebral Perfusion in Total Aortic Arch Replacement. Ann Thorac Surg 2018;105:54-61.

28. Leshnower BG, Myung RJ, Kilgo PD, et al. Moderate hypothermia and unilateral selective antegrade cerebral perfusion: A contemporary cerebral protection strategy for aortic arch surgery. Ann Thorac Surg 2010;90:547-54.

29. Leshnower BG, Rangaraju S, Allen JW, et al. Deep Hypothermia With Retrograde Cerebral Perfusion Versus Moderate Hypothermia With Antegrade Cerebral Perfusion for Arch Surgery. Ann Thorac Surg 2019;107:1104-10.

30. Kamiya H, Hagl C, Kropivnitskaya I, et al. The safety of moderate hypothermic lower body circulatory arrest with selective cerebral perfusion: A propensity score analysis. J Thorac Cardiovasc Surg 2007;133:501-9.
31. Vallabhajosyula P, Jassar AS, Menon RS, et al. Moderate versus deep hypothermic circulatory arrest for elective aortic transverse hemiarch reconstruction. Ann Thorac Surg 2015;99:1511-7.

32. Poon SS, Estrera A, Oo A, et al. Is moderate hypothermic circulatory arrest with selective antegrade cerebral perfusion superior to deep hypothermic circulatory arrest in elective aortic arch surgery? Interact Cardiovasc Thorac Surg 2016;23:462-8.

33. El-Sayed Ahmad A, Papadopoulos N, Risteski P, et al. The Standardized Concept of Moderate-to-Mild $\left(\geq 28^{\circ} \mathrm{C}\right)$ Systemic Hypothermia During Selective Antegrade Cerebral Perfusion for All-Comers in Aortic Arch Surgery: Single-Center Experience in 587 Consecutive Patients Over a 15-Year Period. Ann Thorac Surg 2017;104:49-55.

34. Urbanski PP, Lenos A, Bougioukakis P, et al. Mildto-moderate hypothermia in aortic arch surgery using circulatory arrest: A change of paradigm? Eur J Cardiothorac Surg 2012;41:185-91.

35. Hameed I, Rahouma M, Khan FM, et al. Cerebral protection strategies in aortic arch surgery: A network meta-analysis. J Thorac Cardiovasc Surg 2019;S00225223(19)30483-0.

36. Okita Y, Miyata H, Motomura N, et al. A study of brain protection during total arch replacement comparing antegrade cerebral perfusion versus hypothermic circulatory arrest, with or without retrograde cerebral perfusion: Analysis based on the Japan Adult Cardiovascular Surgery Database. J Thorac Cardiovasc Surg 2015;149:S65-73.

37. Englum BR, He X, Gulack BC, et al. Hypothermia and cerebral protection strategies in aortic arch surgery: a comparative effectiveness analysis from the STS Adult Cardiac Surgery Database. Eur J Cardiothorac Surg 2017;52:492-8.

38. Fan S, Li H, Wang D, et al. Effects of four major brain protection strategies during proximal aortic surgery: A systematic review and network meta-analysis. Int J Surg 2019;63:8-15.

39. Tian DH, Weller J, Hasmat S, et al. Adjunct retrograde cerebral perfusion provides superior outcomes compared with hypothermic circulatory arrest alone: A meta-analysis. J Thorac Cardiovasc Surg 2018;156:1339-1348.e7.

40. Apostolakis E, Akinosoglou K. The methodologies of hypothermic circulatory arrest and of antegrade and retrograde cerebral perfusion for aortic arch surgery. Ann Thorac Cardiovasc Surg 2008;14:138-48.

41. Augoustides JGT, Andritsos M. Innovations in Aortic 
Disease: The Ascending Aorta and Aortic Arch. J

Cardiothorac Vasc Anesth 2010;24:198-207.

42. Lau C, Gaudino M, Iannacone EM, et al. Retrograde Cerebral Perfusion Is Effective for Prolonged Circulatory Arrest in Arch Aneurysm Repair. Ann Thorac Surg 2018;105:491-7.

43. Hu Z, Wang Z, Ren Z, et al. Similar cerebral protective effectiveness of antegrade and retrograde cerebral perfusion combined with deep hypothermia circulatory arrest in aortic arch surgery: A meta-analysis and systematic review of 5060 patients. J Thorac Cardiovasc Surg 2014;148:544-60.

44. Filgueiras CL, Winsborrow BA, Ye J, et al. A 31P-magnetic resonance study of antegrade and retrograde cerebral perfusion during aortic arch surgery in pigs. J Thorac Cardiovasc Surg 1995;110:55-62.

45. Filgueiras CL, Ryner L, Ye J, et al. Cerebral protection during moderate hypothermic circulatory arrest: Histopathology and magnetic resonance spectroscopy of brain energetics and intracellular $\mathrm{pH}$ in pigs. J Thorac Cardiovasc Surg 1996;112:1073-80.

46. Hage A, Stevens LM, Ouzounian M, et al. Impact of brain protection strategies on mortality and stroke in patients undergoing aortic arch repair with hypothermic circulatory arrest: evidence from the Canadian Thoracic Aortic Collaborative. Eur J Cardiothorac Surg 2020. [Epub ahead of print].

47. Angeloni E, Melina G, Refice SK, et al. Unilateral versus bilateral antegrade cerebral protection during aortic surgery: An updated meta-analysis. Ann Thorac Surg 2015;99:2024-31.

48. Spielvogel D, Kai M, Tang GHL, et al. Selective cerebral perfusion: A review of the evidence. J Thorac Cardiovasc Surg 2013;145:S59-62.

49. Tong G, Zhang B, Zhou X, et al. Bilateral versus unilateral antegrade cerebral perfusion in total arch replacement for type A aortic dissection. J Thorac Cardiovasc Surg 2017;154:767-75.

50. Tang GHL, Kai M, Malekan R, et al. Trifurcated graft replacement of the aortic arch: State of the art. J Thorac Cardiovasc Surg 2015;149:S55-8.

51. Spielvogel D, Strauch JT, Minanov OP, et al. Aortic arch replacement using a trifurcated graft and selective cerebral antegrade perfusion. Ann Thorac Surg 2002;74:S1810-4; discussion S1825-32.

52. Yokawa K, Ikeno Y, Henmi S, et al. Impact of shaggy aorta on outcomes of open thoracoabdominal aortic aneurysm repair. J Thorac Cardiovasc Surg 2019. [Epub ahead of print].

53. Sultan I, McGarvey J, Vallabhajosyula P, et al. Routine use of hemiarch during acute type A aortic dissection repair. Ann Cardiothorac Surg 2016;5:245-7.

54. Di Marco L, Leone A, Murana G, et al. Acute type A aortic dissection: Rationale and outcomes of extensive repair of the arch and distal aorta. Int J Cardiol 2018;267:145-9.

55. Ouzounian M, David TE. Total aortic repair for acute type A dissection: Not every patient;not every surgeon. J Thorac Cardiovasc Surg 2019;157:1-2.

56. Girardi LN. Is a more extensive operation justified for acute type A dissection repair? J Thorac Cardiovasc Surg 2019;157:12-3.

57. Trivedi D, Navid F, Balzer JR, et al. Aggressive aortic arch and carotid replacement strategy for type a aortic dissection improves neurologic outcomes presented at the fifty-first annual meeting of the society of thoracic surgeons, San Diego, CA, Jan 24-28, 2015. Ann Thorac Surg 2016;101:896-903.

58. Westaby S, Saito S, Katsumata T. Acute type A dissection: Conservative methods provide consistently low mortality. Ann Thorac Surg 2002;73:707-13.

59. Di Eusanio M, Berretta P, Cefarelli M, et al. Total Arch Replacement Versus More Conservative Management in Type A Acute Aortic Dissection. Ann Thorac Surg 2015;100:88-94.

60. Poon SS, Theologou T, Harrington D, et al. Hemiarch versus total aortic arch replacement in acute type A dissection: A systematic review and meta-analysis. Ann Cardiothorac Surg 2016;5:156-73.

61. Matalanis G, Perera NK, Galvin SD. Total aortic repair: The new paradigm in the treatment of acute type A aortic dissection. Ann Cardiothorac Surg 2016;5:216-21.

62. Matalanis G, Ip S. A new paradigm in the management of acute type A aortic dissection: Total aortic repair. J Thorac Cardiovasc Surg 2019;157:3-11.

63. Sultan I, Szeto WY. Decision making in acute DeBakey i aortic dissection: Balancing extensive arch reconstruction versus mortality. J Thorac Cardiovasc Surg 2016;151:349-50.

64. Bin Mahmood SU, Mori M, Geirsson A, et al. Acute Type A Aortic Dissection Surgery Performed by Aortic Specialists Improves 2-Year Outcomes. Aorta 2019;7:1-6.

65. Waterford SD, Gardner RL, Moon MR. Extent of Aortic Replacement in Type A Dissection: Current Answers for an Endless Debate. Ann Thorac Surg 2018;106:1246-50.

66. Eldeiry M, Aftab M, Bergeron E, et al. The Buffalo Trunk Technique for Aortic Arch Reconstruction. Ann Thorac 
Surg 2019;108:680-6.

67. Chiu P, Trojan J, Tsou S, et al. Limited root repair in acute type A aortic dissection is safe but results in increased risk of reoperation. J Thorac Cardiovasc Surg 2018;155:1-7.e1.

68. Schäfers HJ. Root replacement in acute dissection type A-A superior procedure? J Thorac Cardiovasc Surg 2018;155:8-9.

69. Li J, Sun Y, Zhou T, et al. David reimplantation with simultaneous total arch replacement and stented elephant trunk for acute type A aortic dissection. J Thorac Cardiovasc Surg 2020. [Epub ahead of print].

70. Yang B, Patel HJ, Sorek C, et al. Sixteen-Year Experience of David and Bentall Procedures in Acute Type A Aortic Dissection. Ann Thorac Surg 2018;105:779-84.

71. Rosenblum JM, Leshnower BG, Moon RC, et al. Durability and safety of David V valve-sparing root replacement in acute type A aortic dissection. J Thorac Cardiovasc Surg 2019;157:14-23.e1.

72. Wu J, Huang Y, Qiu J, et al. Is valve-sparing root replacement a safe option in acute type A aortic dissection? A systematic review and meta-analysis. Interact Cardiovasc Thorac Surg 2019;29:766-75.

73. Sievers HH, Richardt D, Diwoky M, et al. Survival and reoperation after valve-sparing root replacement and root repair in acute type A dissection. J Thorac Cardiovasc Surg 2018;156:2076-2082.e2.

74. Levy G, DeAnda A. Valve-sparing root replacement in type A dissection: Age and experience matter. J Thorac Cardiovasc Surg 2019;157:24-5.

75. Yang B, Norton EL, Hobbs R, et al. Short- and longterm outcomes of aortic root repair and replacement in patients undergoing acute type A aortic dissection repair: Twenty-year experience. J Thorac Cardiovasc Surg 2019;157:2125-36.

76. Qiu J, Wu J, Xie E, et al. Surgical management and outcomes of aortic root in acute type A Aortic Dissection. Ann Thorac Surg 2019. [Epub ahead of print].

77. Bergeron EJ, Mosca MS, Aftab M, et al. Neuroprotection Strategies in Aortic Surgery. Cardiol Clin 2017;35:453-65.

doi: $10.21037 /$ jovs-20-82

Cite this article as: Ghincea CV, Aftab M, Ikeno Y, Mesher AL, Reece TB. Cerebral protection in type A aortic dissection. J Vis Surg 2021;7:41.
78. Zanatta P, Benvenuti SM, Bosco E, et al. Multimodal brain monitoring reduces major neurologic complications in cardiac surgery. J Cardiothorac Vasc Anesth 2011;25:1076-85.

79. Fedorow C, Grocott HP. Cerebral monitoring to optimize outcomes after cardiac surgery. Curr Opin Anaesthesiol 2010;23:89-94.

80. Zheng F, Sheinberg R, Yee MS, et al. Cerebral NIRS Monitoring and Neurological Outcomes in Adult Cardiac Surgery Patients and Neurological Outcomes. Anesth Analg 2013;116:1-28.

81. Tanaka Y, Kawaguchi M, Noguchi Y, et al. Systematic review of motor evoked potentials monitoring during thoracic and thoracoabdominal aortic aneurysm open repair surgery: a diagnostic meta-analysis. J Anesth 2016;30:1037-50.

82. Ghincea C V., Anderson DA, Ikeno Y, et al. Utility of neuromonitoring in hypothermic circulatory arrest cases for early detection of stroke: Listening through the noise. J Thorac Cardiovasc Surg 2020. [Epub ahead of print].

83. Wilkey BJ, Weitzel NS. Anesthetic Considerations for Surgery on the Aortic Arch. Semin Cardiothorac Vasc Anesth 2016;20:265-72.

84. Krüger T, Hoffmann I, Blettner M, et al. Intraoperative neuroprotective drugs without beneficial effects? Results of the german registry for acute aortic dissection type a (GERAADA). Eur J Cardiothorac Surg 2013;44:939-46.

85. Svyatets M, Tolani K, Zhang M, et al. Perioperative management of deep hypothermic circulatory arrest. J Cardiothorac Vasc Anesth 2010;24:644-55.

86. Van Der Starre PJA. Deep hypothermia and circulatory arrest. Thorac Aortic Dis 2006;101-8.

87. Al-Hashimi S, Zaman M, Waterworth P, et al. Does the use of thiopental provide added cerebral protection during deep hypothermic circulatory arrest? Interact Cardiovasc Thorac Surg 2013;17:392-7.

88. Salameh A, Dhein S, Dähnert I, et al. Neuroprotective strategies during cardiac surgery with cardiopulmonary bypass. Int J Mol Sci 2016;17:1945. 